\title{
Enhanced ionization efficiency in TIMS analyses of plutonium and americium using porous ion emitters
}

\author{
Matthew L. Baruzzini ${ }^{\mathrm{a}, \mathrm{b}, *}$, Howard L. Hall ${ }^{\mathrm{b}}$, Matthew G. Watrous ${ }^{\mathrm{c}}$, Khalil \\ J. Spencer ${ }^{\mathrm{a}}$, Floyd E. Stanley ${ }^{\mathrm{a}}$ \\ ${ }^{a}$ Los Alamos National Laboratory, Los Alamos, NM 87545, USA \\ ${ }^{b}$ Department of Nuclear Engineering, University of Tennessee, Knoxville, TN 37996, USA \\ ${ }^{c}$ Idaho National Laboratory, Idaho Falls, ID 83415, USA
}

\begin{abstract}
Investigations of enhanced sample utilization in thermal ionization mass spectrometry (TIMS) using porous ion emitter (PIE) techniques for the analyses of trace quantities of americium and plutonium were performed. Repeat ionization efficiency (i.e., the ratio of ions detected to atoms loaded on the filament) measurements were conducted on sample sizes ranging from 10-100 pg for americium and 1-100 pg for plutonium using PIE and traditional (i.e., a single, zone-refined rhenium, flat filament ribbon with a carbon ionization enhancer) TIMS filament sources. When compared to traditional filaments, PIEs exhibited an average boost in ionization efficiency of $\sim 550 \%$ for plutonium and $\sim 1100 \%$ for americium. A maximum average efficiency of $1.09 \%$ was observed at a $1 \mathrm{pg}$ plutonium sample loading using PIEs. Supplementary trials were conducted using newly developed platinum PIEs to analyze $10 \mathrm{pg}$ mass loadings of plutonium. Platinum PIEs exhibited an additional $\sim 134 \%$ boost in ion yield over standard PIEs and $\sim 736 \%$ over traditional filaments at the same sample loading level.
\end{abstract}

Keywords: Thermal ionization mass spectrometry (TIMS), Porous ion emitter (PIE), Ionization efficiency, Americium, Plutonium, Nuclear forensics

\footnotetext{
${ }^{*}$ Corresponding author

Email address: mbaruzzini@lanl.gov (Matthew L. Baruzzini)
}

Preprint submitted to International Journal of Mass Spectrometry

November 9, 2016

(C) 2016. This manuscript version is made available under the Elsevier user license http://www.elsevier.com/open-access/userlicense/1.0/ 


\section{Introduction}

TIMS has long been the method of choice for the isotopic ratio determination of nuclear materials and is widely considered the gold standard for such analyses. However, traditional filament loading techniques are associated with ionization 5 efficiencies of less than $0.1 \%$ for actinides [1]; the majority of the sample is not measured. Such low efficiencies significantly limit the ability make accurate and precise measurements of high priority elements (e.g., americium and plutonium) that could potentially provide critical signatures in nuclear forensic, non-proliferation and safeguards efforts.

For decades, the TIMS resin bead technique has proven to be one of the most effective methods for the analysis of actinide samples. However, despite several advantages, the resin bead approach is still hindered by long preparation time and tediousness of securing a pre-loaded bead atop the filament increasing the chance of losing a valuable sample. More recently, the use of thermal ion cavity (TIC) sources have been proposed as a method to improve sample utilization. While enhancements in ionization efficiency have been demonstrated using (TIC), there are significant drawbacks to implementing this method; namely, costly instrument modifications and sample dedication [2].

PIEs have been proposed as an alternative approach for boosting ion yields.

Theses sources have demonstrated significant enhancements in ionization efficiency during the analysis of trace quantities of uranium [3] and thorium 44. Unlike TIC sources, PIEs can be implemented with no modifications to the mass spectrometer. Proposed mechanisms of improved sample utilization associated with the use of PIE sources include increased ionizing surface contact area, superior ion optical properties, and bombardment by ions emitted by the PIE material itself 4. Neutral atoms incur multiple interactions with the ionizing surface as they traverse the PIE structure thus limiting their rapid escape, increasing the probability of ionization. PIEs have been shown to eliminate sample migration across the filament; as a result, they exhibit superior abundance sensitivity when compared to multi-filament and resin bead techniques [3]. 
Concentrating the sample at the center of a filament is essential for maximizing ion beam focusing and transmission, as magnetic sector instruments are very sensitive to the location of ion production on a filament. Previous research suggests that additional ions may be generated via collisions between escaped neutral atoms with platinum ions emitted as the PIE material is consumed during heating 4]. The extent to which each mechanism contributes to the overall boost in ionization efficiency is unknown at this time.

\section{Experimental methods and equipment}

\subsection{Filament pre-treatment}

40

Prior to the addition of any ionization enhancers, all filaments used during this experiment were pre-treated in a GV Instruments Ltd. (now Isotopx: Middlewich, Cheshire; UK) filament bake-out unit evacuated to $\sim 10^{-7}$ mbar $\left(\sim 10^{-5} \mathrm{~Pa}\right)$. The filaments were slowly heated to $4.5 \mathrm{~A}$ and held at this temperature for roughly ten minutes to remove any impurities (e.g., hydrocarbons, environmental uranium, etc.) that may be present in or on the filament ribbons. Traditional filaments and those intended for use with PIEs were subjected to identical heating and cooling conditions prior to sample loading.

\subsubsection{Traditional filament preparation}

Traditional filaments were prepared by pipetting $1 \mu \mathrm{L}$ of a colloidal graphite solution (Ultra Carbon Corporation; Bay City, MI) atop each zone-refined rhenium filament ribbon (H. Cross Co.; Moonachie, NJ). Extreme care was taken to localize the graphite solution to the center of the filament surface during application. The graphite solution was partially dried via resistive heating at 1 A for approximately fifteen seconds.

\subsubsection{PIE stock preparation}

All PIE stock material used herein was prepared in-house, at Los Alamos National Laboratory (LANL). Standard PIE stock was made by combining equal parts, by mass, of platinum powder (325 mesh, Sigma Aldritch; St. Louis, MO), 


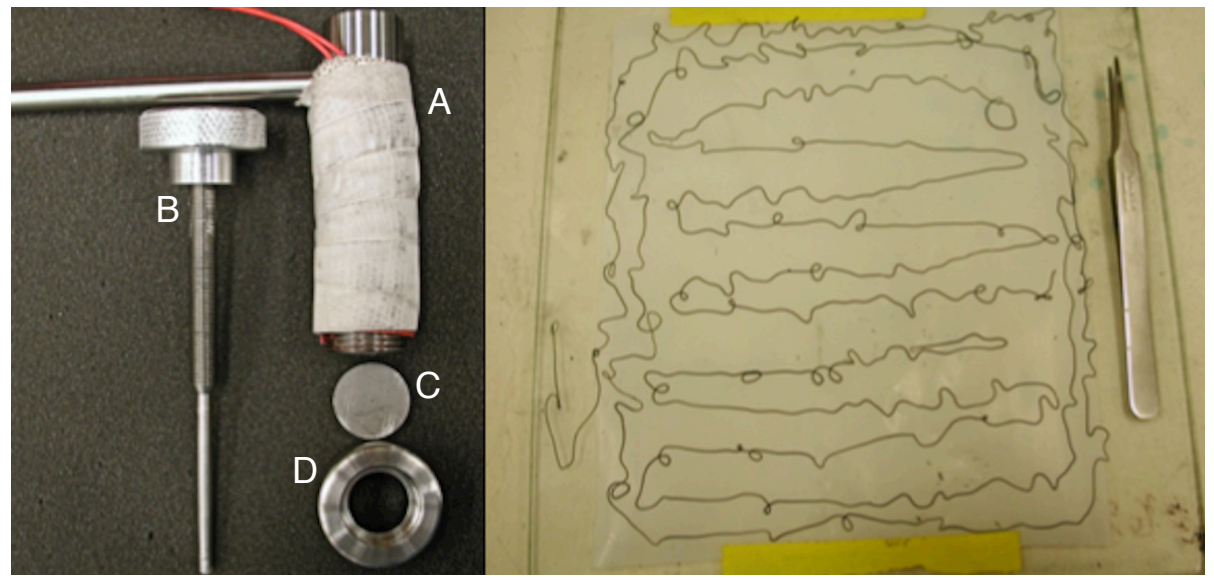

Figure 1: Photographs of the custom fabricated extruder, on the left, and a PIE rope extruded onto a quartz glass plate, on the right. The PIE stock material is loaded in the extruder body (A) which is warmed via heat tape; the temperature is controlled using a Variac. The threaded plunger (B) is slowly turned, forcing the stock material through a small conical hole in the die $(\mathrm{C})$; the die is held in place with a threaded ring (D).

rhenium powder (325 mesh, Sigma Aldritch; St. Louis, MO), and a hot melt gluing agent (not well characterized). The hot glue was melted atop a quartz glass microscope slide using a laboratory hot plate; the platinum and rhenium powders were added to the melted hot glue and thoroughly mixed. Stock material for platinum PIEs was fabricated by incorporating platinum powder (325-mesh, Sigma Aldritch; St. Louis, MO) and hot melt glue in a two-to-one ratio, by mass. The platinum powder and glue were amalgamated in the manner previously described for standard PIE material. A custom built extruder was used to reproducibly expel each PIE stock material into a small, $\sim 550 \mu \mathrm{m}$, diameter rope. Images of the extruder and a PIE rope atop a quartz glass plate are presented in Figure1.

\subsubsection{Preparation of PIE filaments}

Sections of PIE stock material, approximately $100 \mu \mathrm{m}$ in height, were secured to the center of each outgassed rhenium filament ribbon via gentle heating at $1 \mathrm{~A}$ for approximately five seconds. The filaments were then placed in the bake-out 
unit where the PIE stock was sintered; the result is a three-dimensional porous bead $\sim 700 \mu \mathrm{m}$ diameter and $\sim 75 \mu \mathrm{m}$ in height. Details of the sintering process of platinum/rhenium (Pt/Re) PIE and platinum PIE filaments are provided below.

Pt/Re PIEs. The filaments were loaded in the filament bake-out unit, placed under vacuum, $\sim 10^{-7}$ mbar $\left(\sim 10^{-5} \mathrm{~Pa}\right)$, and slowly heated to $\sim 1700^{\circ} \mathrm{C}$; the filaments were held at for this temperature for twenty minutes. The baking process serves two purposes; 1) to volatilize the glue and 2) sinter the PIE to itself and to the filament surface. The pressure in the bake-out unit was carefully monitored to ensure that there were no pressure spikes as the glue volatilized during the sintering process. Rapid volatilization of the glue could result in the 85 PIE blowing-off the prior to fusing itself to the filament or the in formation of large pores in the PIE structure. Explicit details for preparation of Pt/Re PIEs can be found in reference [5].

Platinum PIEs. Filaments were mounted in the filament bake-out unit which was then evacuated to $\sim 10^{-7} \mathrm{mbar}\left(\sim 10^{-5} \mathrm{~Pa}\right)$. Attempts at making platinum 90 PIEs using the heating method described in 2.1.3 resulted in failure; either the PIE material was melted into a solid, non-porous, mass of platinum or was completely lost due to being volatilized from the filament surface. The most desirable results were produced by gradually heating the filaments at a rate of $0.25 \mathrm{~A} \mathrm{~min}^{-1}$ until reaching a temperature of $\sim 1450{ }^{\circ} \mathrm{C}$. Filaments were held at 95 this temperature for approximately ten minutes.

Prior to sample loading, two drops of poly(4-styrenesulfonic acid) cation exchange polymer $\left(\mathrm{M}_{W}=75000,18 \mathrm{wt} \%\right.$ in $\mathrm{H}_{2} \mathrm{O}$ : Sigma Aldritch; St. Louis, $\left.\mathrm{MO}\right)$, diluted in $18 \mathrm{M} \Omega$ deionized (DI) water to a concentration of approximately $3 \%$ by mass, were added to each PIE and dried by heating at $1 \mathrm{~A}$ for approximately fifteen seconds. The ion exchange resin was added in an effort to enhance sample incorporation into the PIE. 
In an effort to limit systematic errors that may be introduced by irregularities in PIE construction, great care was taken to select a set of filaments exhibiting similar characteristics. Each PIE filament was examined using an optical microscope; criterion used in filament selection included diameter, height, and consistency in pore size (i.e., no large cavities created as the hot glue was volatilized) of the PIE.

\subsection{Sample application}

Americium stock for sampling was prepared from a well-characterized ${ }^{243} \mathrm{Am}$ spike; Certified Reference Material (CRM)-144 (New Brunswick Laboratory (NBL); Chicago, IL), was used to prepare the stock plutonium solution. Aliquots of each stock solution were prepared in concentrations ranging from 2-200 partsper-billion $\left(\mathrm{ng} \mathrm{g}^{-1}\right)$ using Optima ${ }^{T M}$ grade nitric acid (Fisher Scientific, Pittsburgh, PA; USA ) diluted to $1 \mathrm{~mol} \mathrm{~L}^{-1}$ using $18 \mathrm{M} \Omega$ DI water to limit loading volume to $0.5 \mu \mathrm{L}$. The small aliquot size allowed for precise analyte application atop the filaments as well as minimizing sample diffusion across the filament. Samples were deposited directly onto the PIE or atop the damp colloidal graphite solution using a $2.5 \mu \mathrm{L}$ capacity pipette and dried completely in preparation for subsequent analysis.

\subsection{TIMS instrumentation}

All sample utilization measurements were made using an Isoprobe- $T^{\text {TM }}$ from GV Instruments Ltd. (now Isotopx: Middlewich, Cheshire; UK) at LANL. The ion collection system consists of nine, variable-position, Faraday cup detectors equipped with $10^{11} \Omega$ resistors and a static ion counting Daly detector at the axial position mounted behind a wide aperture retarding potential (WARP) energy filter. Mass spectrometer performance was controlled via the GV Instruments IonVantage software package installed on a Dell Optiplex PC (Rock Round, TX; USA). To ensure maximum measurement precision, the mass spectrometer was warmed for at least an hour under electronic conditions similar 
to those employed during sample analysis. Following instrument warm-up, primary instrument tuning (e.g., ion optic lens, peak shape, and peak centering adjustments) was conducted using an outgassed bare zone-refined rhenium filament. Fine-tuning was conducted at the mass number of interest before the analysis of each sample. Due to the long runtime of PIE equipped filaments, additional fine-tunings were conducted, as needed, during sample analysis to compensate for any drift in electronics that may occur. Pressure in the source housing was kept as low as possible, $\sim 10^{-8}$ mbar $\left(\sim 10^{-6} \mathrm{~Pa}\right)$, with the aid of liquid nitrogen added to the cold trap as needed.

\section{Results and discussion}

Side-by-side ionization efficiency measurements were conducted using $\mathrm{Pt} / \mathrm{Re}$ PIE, platinum PIE, and standard single rhenium filament assemblies. Sample ion beams were monitored exclusively using the Daly detector in conjunction with the WARP filter. Integrated signals were determined off-line, post-analysis and used to calculate ionization efficiencies. All samples were run to signal exhaustion, approximately $500 \mathrm{cps}$.

\subsection{Americium ionization efficiency}

Repeat sample utilization measurements were conducted at $10 \mathrm{pg}, 50 \mathrm{pg}$, and $100 \mathrm{pg}$ mass loading levels of americium. An average ${ }^{243} \mathrm{Am}^{+}$ion yield of $0.81 \%$ was achieved using Pt/Re PIE equipped filaments compared to $0.07 \%$ using traditional single filament assemblies; this translates to an $\sim 1100 \%$ enhancement in sample utilization at all loading levels tested. Additionally, PIEs exhibit superior ion yield when compared to published ionization efficiency values for americium using both the resin bead and TIC techniques. Bürger et al. [1] report average efficiencies of $0.16 \%$ for the resin bead method and $0.63 \%$ for resin bead loads with carbon additive in rhenium cavities at loading levels of $0.17-0.29 \mathrm{pg}$ and $0.29 \mathrm{pg}$, respectively. Data for individual trials are plotted in Figure 2 the shaded areas indicate theoretical americium ionization efficiencies, 


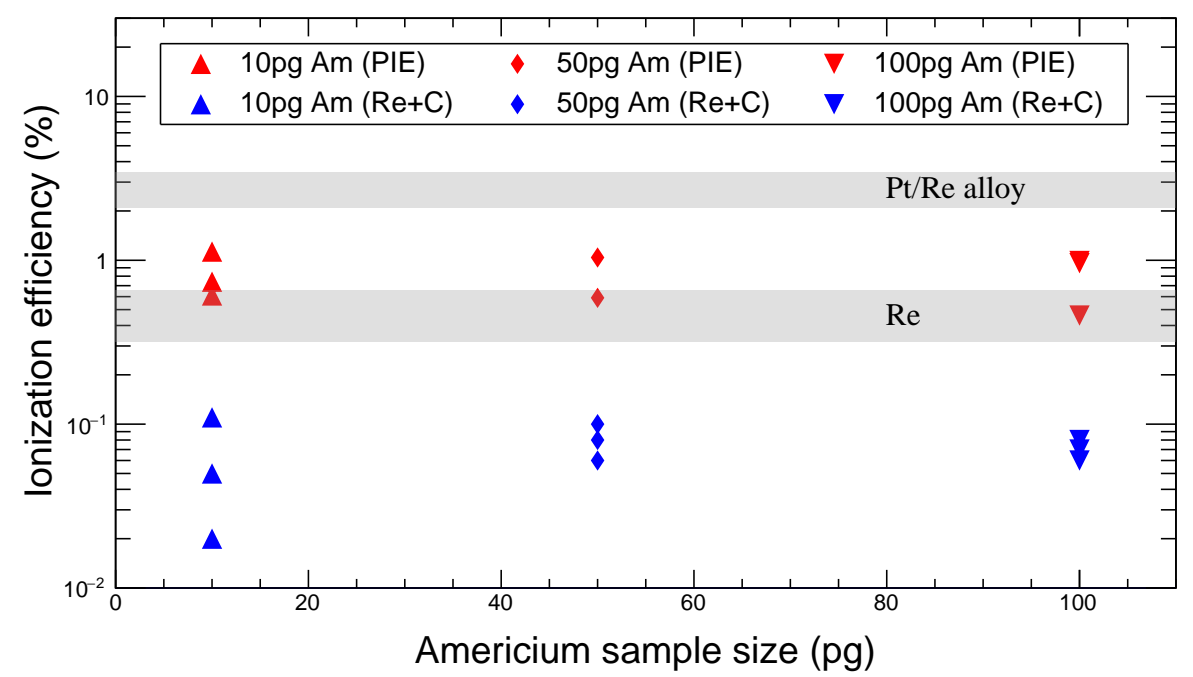

Figure 2: Americium ionization efficiency data acquired using Pt/Re PIE and traditional single rhenium filaments. The shaded areas indicate theoretical americium ionization efficiencies atop rhenium and Pt/Re alloy filaments.

as predicted by the Saha-Langmuir equation;

$$
\frac{n^{+}}{n^{0}} \propto \exp \left[\frac{\phi-\Delta E_{I}}{k T}\right],
$$

using standard polycrystalline rhenium filaments and filaments made from a $\mathrm{Pt} /$ Re alloy. Here $\phi, T$ are the work function (in $\mathrm{eV}$ ) and temperature (in $\mathrm{K}$ ) of the ionizing surface; $\Delta E_{I}$ is the first ionization potential (in $\mathrm{eV}$ ) of the element to be analyzed, $k$ is the Boltzmann constant, $8.6173303(50) \times 10^{-5} \mathrm{eV} \mathrm{K}^{-1}[\underline{6}$. A filament temperature range of $2024-2324 \mathrm{~K}$, rhenium work function of $4.98 \mathrm{eV}$ [2], americium first ionization potential of $5.9738(2) \mathrm{eV}$ [7, and Pt/Re alloy $(50 \%$ concentration of platinum) work function of $5.30 \mathrm{eV}$ were used for calculating theoretical ion yields. The Pt/Re alloy work function was theoretically determined using the methods described in reference [8]; the work function of the alloy was used to predict performance of Pt/Re PIEs, which are a mechanical mixture of platinum and rhenium and are not a true alloy. Figure 2 clearly illustrates that Pt/Re PIEs consistently produced americium ion yields exceeding that predicted by Equation 1 for thermal ionization using conventional, 
Table 1: A summary of average americium ionization efficiency data collected using PIE and traditional filaments as ion sources.

\begin{tabular}{lccc}
\hline \multirow{2}{*}{$\begin{array}{l}\text { Am sample } \\
\text { size }(\mathrm{pg})\end{array}$} & \multicolumn{2}{c}{ Ionization Efficiency $(\%)$} & $\begin{array}{r}\text { Boost } \\
\text { Traditional }\end{array}$ \\
\cline { 2 - 3 } 100 & 0.07 & PIE & $\sim 1143$ \\
50 & 0.08 & 0.80 & $\sim 1013$ \\
10 & 0.07 & 0.81 & $\sim 1186$ \\
\hline
\end{tabular}

flat polycrystalline rhenium filaments. Average americium ionization efficiency data are presented in Table 1. A tendency of increased ionization efficiency with decreased sample size was not observed in data acquired over this mass loading range, a commonly observed phenomenon attributed to analyte atoms competing for filament surface area [2, 9. However, this trend may become evident if analyses were conducted over broader range of sample sizes. At $50 \mathrm{pg}$ and $100 \mathrm{pg}$ sample mass loading levels both standard and PIE filaments were able to produce stable ion beams; however, at $10 \mathrm{pg}$ loading levels traditional filaments were unable to maintain aiming intensity; traditional filaments were run at aiming intensities ranging from $40000-100000 \mathrm{cps}$, PIEs were analyzed at ion beam intensities of $120000 \mathrm{cps}$ and $300000 \mathrm{cps}$. Figure 3 shows that, for $10 \mathrm{pg}$ americium sample loadings, PIEs exhibit greater ability to generate a stable ion beam at a much higher intensity than did their traditional filament equivalents. Inspection of Figure 3 also reveals that PIEs were able to generate a stable ion beam much more quickly (i.e., at a lower temperature) than traditional filaments; we attribute this to the higher work function of PIEs, through the addition of platinum, as well as a substantial increase in surface area associated with PIE sources. The larger surface area increases the probability that evaporated neutral atoms will be ionized as they migrate through the porous structure by promoting multiple interactions with the PIE; this proves significant at lower temperatures where ionization is less likely to occur. Intensity curves associated with PIEs seem to exhibit more "noise" than the traditional filament intensity curves for $10 \mathrm{pg}$ americium sample loadings, as illustrated In 


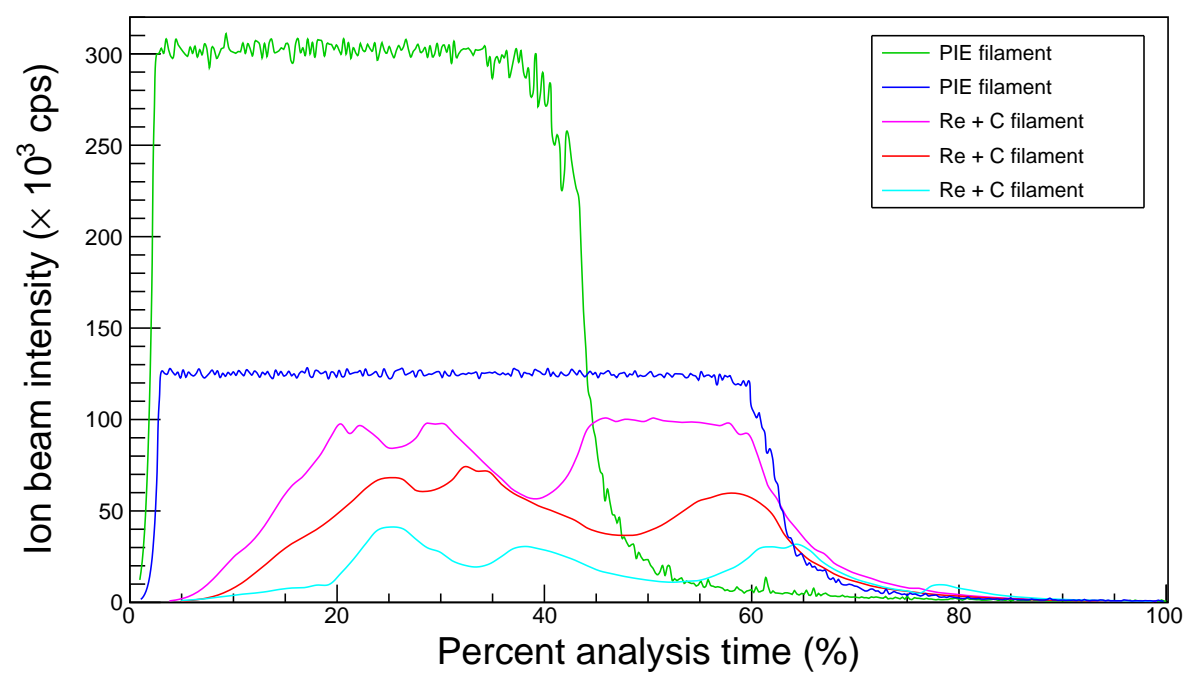

Figure 3: Ion beam intensity as a function of percent runtime representative of PIE and traditional filaments at a $10 \mathrm{pg}$ americium loading level.

Figure 3. This noise was originally thought to be a result of the changing morphology of the PIE as it disintegrates during heating; however, analysis of ion beam intensity curves for larger americium samples reveled that this noise is present in intensity curves of PIE and traditional filaments. Figure 4 shows the signal noise in plateau region of aiming intensity curves for $100 \mathrm{pg}$ americium samples acquired using PIE and traditional filament sources.

\subsection{Plutonium ionization efficiency}

Plutonium ionization efficiency measurements were performed on sample mass loadings of $1 \mathrm{pg}, 10 \mathrm{pg}, 50 \mathrm{pg}$, and $100 \mathrm{pg}$; multiple trials $(N=3-11)$ were conducted at each sample size. The ${ }^{242} \mathrm{Pu}^{+}$ion beam was monitored for these measurements. Ion yield was relatively constant for plutonium mass loadings ranging from 10-100 pg using Pt/Re PIE sources, with an average efficiency of $0.83 \%$. This translates to an approximate $460 \%$ improvement over traditional filaments and a roughly $154 \%$ increase over ionization efficiencies reported for the resin bead technique [1. A maximum average sample utilization of $1.09 \%$ was realized at a $1 \mathrm{pg}$ mass loading on Pt/Re PIEs. The ionization efficiency at 


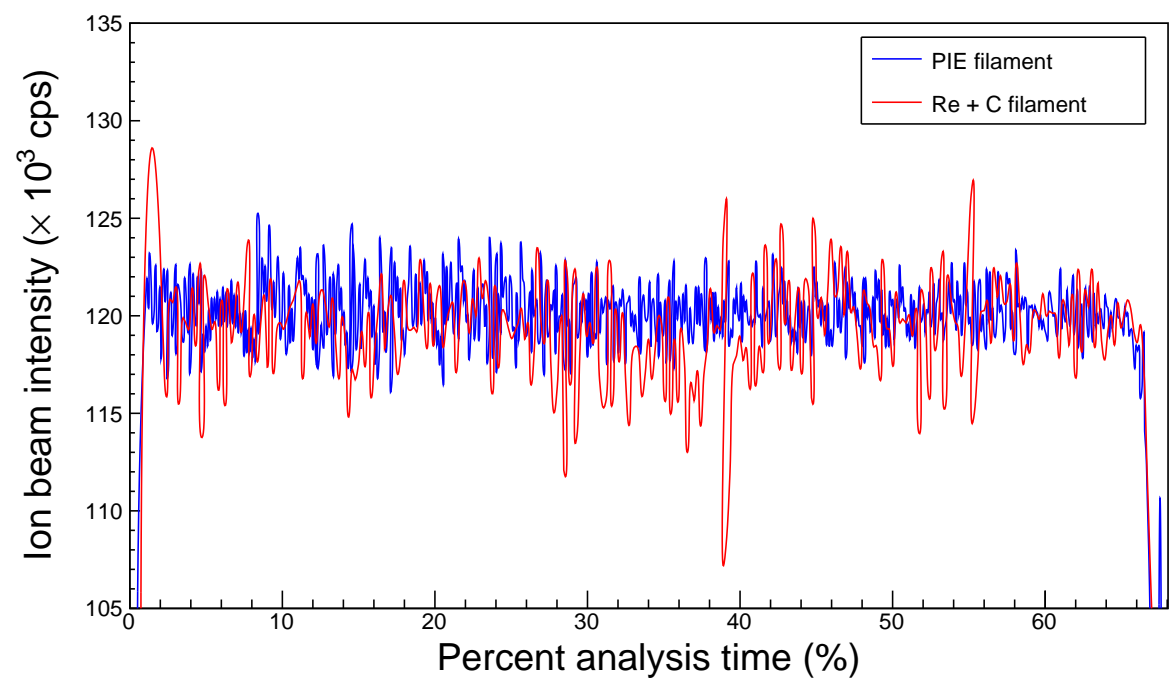

Figure 4: Plateau region of ion beam intensities, as a function of percent runtime, representative of PIE and traditional filaments at a $100 \mathrm{pg}$ americium loading level.

the $1 \mathrm{pg}$ plutonium sample loading achieved during this study employing PIEs is a marked improvement over utilization efficiency of $0.23 \%$ for $0.1 \mathrm{pg}$ mass loading of plutonium reported by [10. Data for trials at 1 pg plutonium sample loadings using traditional filament is not presented as each filament failed rapidly. Data presented in Figure 5 suggests a possible trend of enhanced sample utilization with decreased sample size beginning at the $1 \mathrm{pg}$ sample size; however, additional trials conducted at sample loading levels less than $1 \mathrm{pg}$ are required to confirm this hypothesis. The shaded areas in Figure 5 indicate theoretical plutonium ionization efficiencies atop rhenium and rhenium with carbon additive predicted using Equation 1] a first ionization energy of $6.0260 \mathrm{eV}$ [1] for plutonium, work function values of $4.98 \mathrm{eV}$ and $5.36 \mathrm{eV}$ [2] for rhenium and carburized rhenium, respectively, and a filament temperature range of 2024-2324 K were used. As with americium, PIEs were able to produce plutonium ion yields that routinely exceeded those predicted by the Saha-Langmuir equation 1 using standard bare rhenium filaments.

Additional ionization efficiency measurements $(N=3)$ were conducted at 


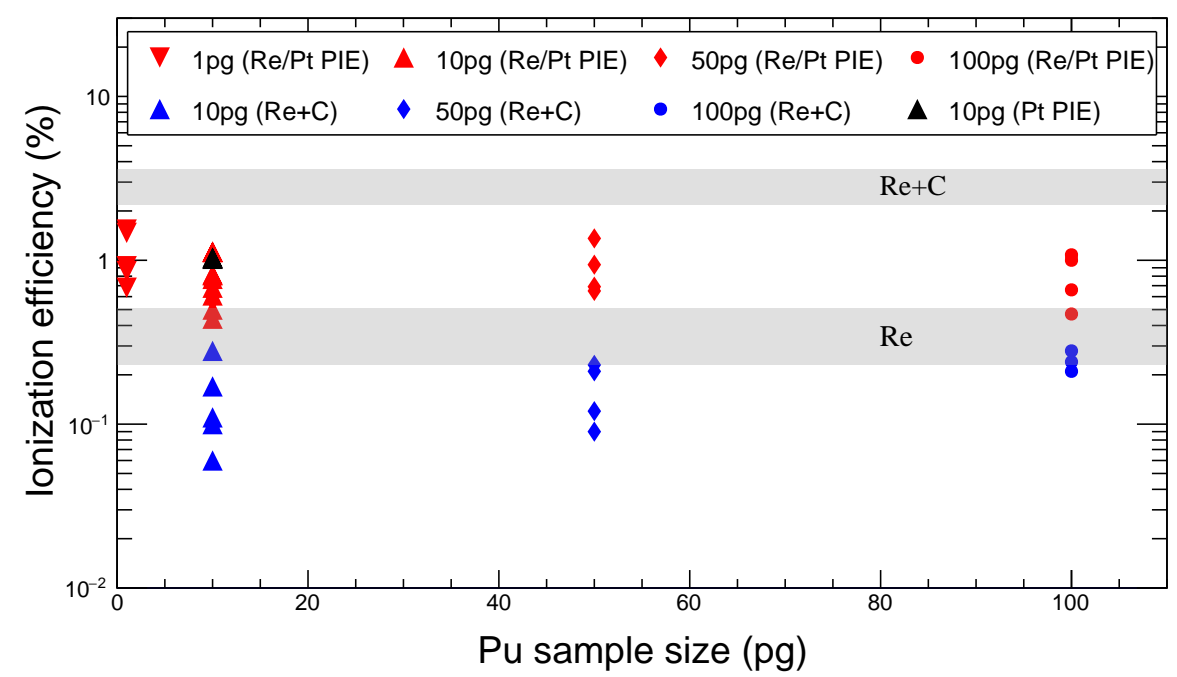

Figure 5: Plutonium ionization efficiency data obtained using Pt/Re PIEs, platinum PIEs, and traditional filaments. The shaded areas indicate theoretical plutonium ionization efficiencies atop bare rhenium and rhenium with a carbon additive.

$10 \mathrm{pg}$ loading levels of plutonium using newly developed PIE sources made completely from platinum. Platinum PIEs demonstrated an additional $134 \%$ increase in sample utilization over their $\mathrm{Pt} / \mathrm{Re}$ counterparts. This boost can likely be attributed to two primary factors: 1) the work function of platinum is higher than that of rhenium [12] and 2) the pores in the platinum PIEs, observed using an optical microscope, appeared to be smaller and more uniform in size than those present in PIEs made from a combination of platinum and rhenium powders. Smaller pores allow for increased contact between the analyte and filament which has proven to be a significant contributor for increased sample utilization associated with PIEs [5]. Figure 6] is a scanning electron microscope (SEM) image illustrating the large pores, 50-100 $\mu \mathrm{m}$ in diameter, found in Pt/Re PIEs. The homogeneity in micro-porous structure of platinum PIEs appears to have the added benefit of increased measurement precision. In this study, percent relative standard deviation (\%RSD) in ionization efficiency measurement results made using platinum PIEs were significantly lower than those made using Pt/Re PIEs or traditional TIMS sources; platinum PIEs exhibited a 


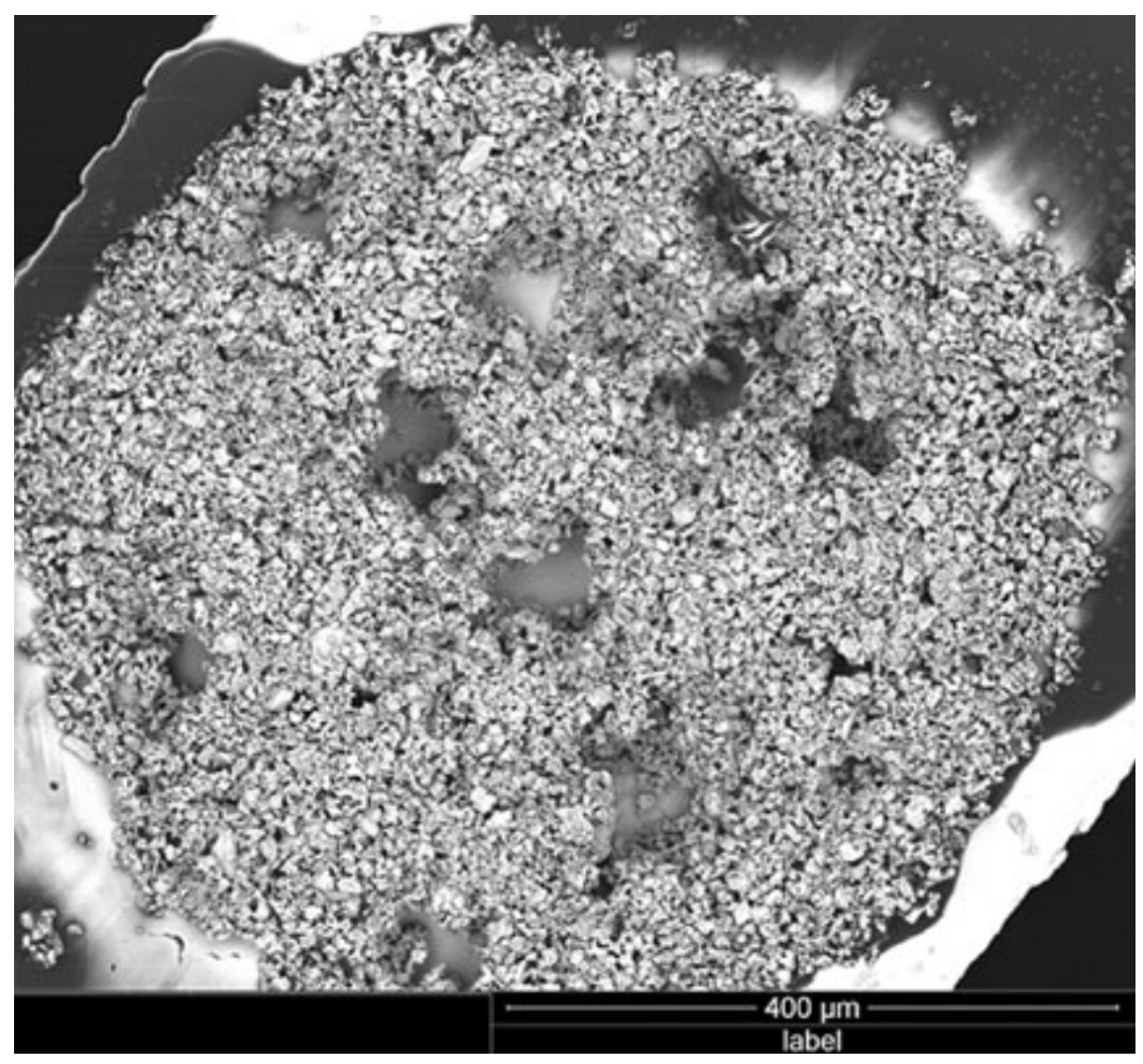

Figure 6: An SEM image of a Pt/Re PIE mounted atop a standard, zone-refined rhenium filament. The larger intermittent pores range in size from $50-100 \mu \mathrm{m}$ in diameter. This figure was adapted from reference [4]. 
Table 2: A summary of average plutonium ionization efficiency data collected using PIE and traditional filaments as ion sources.

\begin{tabular}{lccr}
\hline \multirow{2}{*}{$\begin{array}{l}\text { Pu sample } \\
\text { size }(\mathrm{pg})\end{array}$} & \multicolumn{2}{c}{ Ionization Efficiencies $(\%)$} & $\begin{array}{r}\text { Boost } \\
(\%)\end{array}$ \\
\cline { 2 - 4 } 100 & Traditional & PIE & $\sim 333$ \\
50 & 0.24 & 0.80 & $\sim 569$ \\
10 & 0.16 & 0.91 & $\sim 550$ \\
1 & 0.14 & 0.77 & - \\
10 & - & 1.09 & $\sim 736$ \\
\hline
\end{tabular}

${ }^{a}$ sample analyzed with platinum PIE

measurement precision of $\sim 1 \% \mathrm{RSD}$ whereas ionization efficiency measurements made using Pt/Re PIEs and traditional filaments demonstrated a precision of $\geq 20 \%$ RSD. Averaged ionization efficiency data for each sample mass are listed in Table 2 .

\subsection{Additional considerations}

The extent to which an element can be ionized is highly contingent on the work function of the ionizing surface. This process can be described by the Saha-Langmuir equation (1). Platinum has a higher mean work function than rhenium and should produce ions more easily for a given temperature and analyte. At this point, the work function of platinum-rhenium mechanical mixture has not been studied; however, scanning electron microscope with energy dispersive spectroscopy (SEM/EDS) analyses have shown that platinum-rhenium composition and, as a result, Pt/Re PIE work function are dynamic. During thorium trials the PIEs platinum supply was nearly exhausted within the first fifteen minutes of analyses [4. Due to the highly refractory nature of thorium, TIMS analyses of thorium are conducted at much higher temperatures than those for plutonium. As such, the rate of platinum consumption at lower analysis temperatures is unknown at this time. The increased work function does not seem to be a significant driver in thorium analyses; however, it may prove 
significant in the analysis of actinides with lower ionization potentials such as americium and plutonium.

\section{Conclusions}

This work represents an initial investigation into enhanced ionization efficiency of americium and plutonium using PIEs as TIMS sources. PIE techniques consistently demonstrated substantial improvements in sample utilization during the analyses of americium and plutonium in the 1-100 pg mass loading range when compared to traditional TIMS ion sources. Initial trials, conducted using $10 \mathrm{pg}$ samples of plutonium, suggest that PIEs constructed of $100 \%$ platinum can further enhance ion yield, with greater precision, when compared to $\mathrm{Pt} / \mathrm{Re}$ PIEs. Subsequent investigations of enhanced sample utilization using platinum PIEs, carried out over a broader range of sample sizes and elements, are required to fully explore the efficacy of these sources. Americium ionization efficiency measurements using platinum PIE sources were not investigated during this work.

\section{Acknowledgement}

The authors gratefully acknowledge the support of the U.S. Department of Homeland Security under Grant Award Number, 2012-DN-130-NF0001-02. The views and conclusions contained in this document are those of the authors and should not be interpreted as necessarily representing the official policies, either expressed or implied, of the U.S. Department of Homeland Security. The authors would also to express their gratitude for the support provided by the U.S. Department of Energy/National Nuclear Security Administration Office of Nonproliferation and Verification Research and Development and the U.S. 275 Department of Energy through the LANL/LDRD Program. This document has been reviewed and approved for release under LA-UR-16-26867. 


\section{References}

[1] S. Bürger, L. R. Riciputi, D. A. Bostick, S. Turgeon, E. H. McBay, M. Lavelle, Isotope ratio analysis of actinides, fission products, and geolocators by high-efficiency multi-collector thermal ionization mass spectrometry, International Journal of Mass Spectrometry 286 (2) (2009) 70-82. doi:10.1016/j.ijms.2009.06.010

[2] D. M. Wayne, W. Hang, D. K. McDaniel, R. E. Fields, E. Rios, V. Majidi, The thermal ionization cavity (TIC) source: elucidation of possible mechanisms for enhanced ionization efficiency, International Journal of Mass Spectrometry 216 (1) (2002) 41-57. doi:10.1016/S1387-3806(02) 00551-1.

[3] M. G. Watrous, J. E. Delmore, Measurement of trace uranium isotopes using a porous ion emitter, International Journal of Mass Spectrometry 303 (1) (2011) 1-5. doi:10.1016/j.ijms.2010.11.016.

[4] F. E. Stanley, K. J. Spencer, D. S. Schwartz, M. G. Watrous, J. E. Delmore, Investigating enhanced thorium ionization in TIMS using Re/Pt porous ion emitters, Journal of Radioanalytical and Nuclear Chemistry 299 (3) (2014) 1447-1452. doi:10.1007/s10967-013-2813-3.

[5] M. G. Watrous, J. E. Delmore, M. L. Stone, Porous ion emitters - A new type of thermal ion emitter, International Journal of Mass Spectrometry 296 (1) (2010) 21-24. doi:10.1016/j.ijms.2010.07.015.

[6] NIST, Boltzmann constant in eV/K; http://physics.nist.gov/ cgi-bin/cuu/Value?tkev.

[7] R. Deissenberger, S. Köhler, F. Ames, K. Eberhardt, N. Erdmann, H. Funk, G. Herrmann, H.-J. Kluge, M. Nunnemann, G. Passler, et al., First determination of the ionization potential of americium and curium, Angewandte Chemie International Edition in English 34 (7) (1995) 814-815. doi:10.1002/anie.199508141. 
[8] J. Chrzanowski, Y. Kravtsov, B. Bieg, Application of the work function to study the percentage composition of aluminum alloys, Zeszyty Naukowe/Akademia Morska w Szczecinie 38 (110) (2014) 27-31.

[9] R. L. Edwards, J. H. Chen, G. J. Wasserburg, ${ }^{238} \mathrm{U}-{ }^{234} \mathrm{U}-{ }^{230} \mathrm{Th}-{ }^{232} \mathrm{Th}$ systematics and the precise measurement of time over the past 500,000 years, Earth and Planetary Science Letters 81 (2) (1987) 175-192.

[10] M. G. Watrous, Characterization of the resin bead and development and application of the porous ion emitter for thermal ionization mass spectrometry, Ph.D. thesis, University of Idaho (2010).

[11] NIST, Atomic data for plutonium (Pu); http://physics.nist.gov/ PhysRefData/Handbook/Tables/plutoniumtable1.htm.

[12] H. Kawano, Mean work functions effective for negative-ionic, electronic and positive-ionic emissions from polycrystalline surfaces, Applied Surface Science 252 (14) (2006) 5233-5242. doi:10.1016/j.apsusc.2005.08.005 